\title{
Notch signaling is essential in collecting duct epithelial cell fate determination during development and maintenance of cell type homeostasis in adult
}

\author{
Ming-Zhi Zhang ${ }^{1,2}$ \\ ${ }^{1}$ Division of Nephrology and Hypertension, Department of Medicine, ${ }^{2}$ Vanderbilt Center for Kidney Disease, Vanderbilt University School of \\ Medicine, Nashville, TN, USA \\ Correspondence to: Ming-Zhi Zhang. S3223 Medical Center North, Department of Medicine, Vanderbilt University School of Medicine, Nashville, \\ TN 37232, USA. Email: ming-zhi.zhang@vanderbilt.edu. \\ Provenance: This is an invited article commissioned by the Section Editor Dr. Cheng Yuan, MD, PhD (Zhongnan Hospital, Wuhan University, \\ Wuhan, China). \\ Comment on: Mukherjee M, deRiso J, Otterpohl K, et al. Endogenous Notch Signaling in Adult Kidneys Maintains Segment-Specific Epithelial Cell \\ Types of the Distal Tubules and Collecting Ducts to Ensure Water Homeostasis. J Am Soc Nephrol 2019;30:110-26.
}

Submitted Nov 03, 2019. Accepted for publication Dec 20, 2019.

doi: $10.21037 /$ atm.2019.12.121

View this article at: http://dx.doi.org/10.21037/atm.2019.12.121

Mammalian kidney has at least 26 different cell types (1). The collecting duct derives from the ureteric bud (UB) (2). Nephrogenesis is completed before birth in human beings and mice but continues until postnatal day 11-15 in rats (3). However, renal functional maturation lags behind anatomic maturation in all species. Tubule hypertrophy and hyperplasia and interstitial expansion contribute to the postnatal size increase of the kidney (4). Unlike other segment of nephrons, which contains single epithelial cell type, the collecting duct contains principal cells (PCs) and intercalated cells. PCs and ICs express distinct transporters to enable water and sodium homeostasis and acid-base homeostasis, respectively (5).

PCs express epithelial $\mathrm{Na}^{+}$channel $(\mathrm{ENaC})$ and the water channel Aquaporin $2(\mathrm{AQP} 2)$ at apical membrane and water channels $\mathrm{AQP} 3$ and $\mathrm{AQP} 4$ at basolateral membrane to regulate water and sodium balance. AQP2-positive PCs exist in the connecting tubule and all collecting duct. ICs express V-ATPase and carbonic anhydrase II (CAII) to regulate the acid-base homeostasis. V-ATPase pump protons across the plasma membranes. CAII catalyzes the production of bicarbonate and protons from carbon dioxide and water.

Intercalated cells exist in three forms. A-ICs (or $\alpha$-ICs) are located in connecting tubule and cortical collecting duct and express apical V-ATPase and basolateral anion exchanger 1 (AE1). They are responsible for bicarbonate reabsorption and proton secretion into the urine (5). B-ICs (or $\beta$-ICs) are located in connecting tubule and cortical collecting duct and express apical pendrin (an anion exchanger, $\mathrm{Na}^{+}$-independent $\mathrm{Cl}^{-} / \mathrm{HCO}^{-}$exchanger) and basolateral V-ATPase. They are responsible for secreting bicarbonates into urine and reabsorbing protons. Non-A Non-B ICs express both pendrin and V-ATPase apically to secrete bicarbonates and protons.

In human being, $\mathrm{ENaC}$ mutation with increased activity in PCs causes Liddle syndrome with hypertension and hypokalemia. On the contrary, ENaC mutation with lost function in PCs results in pseudohypoaldosteronism type 1 (PHA1) with hypotension, hyponatremia and hyperkalemia due to excessive loss of sodium. Mutations of either AE1 or V-ATPase in ICs lead to distal renal tubular acidosis (dRTA) due to a failure to acidify urine and decrease plasma bicarbonate. Mutations of AQP2 and vasopressin type 2 receptor cause nephrogenic diabetes insipidus (NDI) due to inability to concentrate urine and polyuria $(6,7)$.

Although the genesis of IC and PC and their relatedness to one another have not been fully understood, progress has been made due to availability of different transgenic mice. The Notch signaling pathway and some transcription factors play an essential in the cell fate differentiation of ICs and PCs. 
Notch signaling mediates a juxtacrine cellular signaling system, in which both the signaling sending cells (with ligands) and the signaling receiving cells (with receptors) are affected by the ligand-receptor crosstalk. Notch receptors (Notch 1-4) are single-pass transmembrane proteins with an extracellular domain, a transmembrane domain and an intracellular domain (NICD). Notch receptors bind ligands in a manner regulated by Deltex and inhibited by NUMB. In mammals, Notch ligands include members of the Delta-like and the Jagged families. With ligand binding, the extracellular domain of Notch is cleaved from the transmembrane-NICD domains by the TACE. The extracellular domain remains bound to the ligand and this complex is endocytosed/recycled within the cells with ligands (signaling sending cells) via ubiquitination by mind bomb (Mib). $\gamma$-secretase releases the NICD from the transmembrane domain of the Notch, allowing its nuclear translocation to regulate the canonical Notch target genes including Myc and the HES-family members.

Adam 10 is a member of a disintegrin and metalloproteinase domain (Adam) family and is able to cleave Notch ligands as well as the proteolytic cleavage of collagen IV, epidermal growth factor, ephrin, and chemokines (8). Adam 10 is expressed in the epithelial cells of collecting duct. Mice with deficient Adam 10 in collecting duct (HoxB7-Cre mice were used) had decreased PC vs $\mathrm{IC}$ ratio in collecting duct due to decreased $\mathrm{PC}$ number and increased IC number, in association with polydipsia, polyuria, and low urine osmolarity (9). Mice with deficient Adam 10 in collecting duct had deficient Notch signaling and increased Foxi1 $1^{+}$ICs in the cortex and medulla (9). Therefore, Adam 10 regulates cell fate determination via activation of Notch signaling, possibly due to regulation of Foxil expression, which occurs before birth.

Mind bomb 1 (Mib1) is an E3 ubiquitin ligase and is important to activate Jag- and Dll-mediated Notch signaling. Mice lacking Mib1 in the collecting duct (HoxB7Cre mice were used) had fewer PCs and corresponding more intercalated cells, in association with polyuria, low urinary osmolality, progressive hydronephrosis, sodium wasting, and NDI (10). Restoration of Notching signaling via transgenic overexpression of Notch intracellular domain led to PCs occupying the entire region (10).

Forkhead box genes are a large family of transcription factors that are essential in cell-type fate during development. Foxi1 is an important transcription factor for intercalated cell differentiation. Blomqvist et al. reported that mice with deficient Foxi1 had collecting duct with single cell type expressing AQP2 (PC marker) and CAII (IC marker) without detectable other intercalated cell markers such as V-ATPase, AE2, AE4 and pendrin and exhibited dRTA. Therefore, Foxil play a critical role in determining IC fate during collecting duct development (11).

Recently, Werth et al. found that Tfcp2I1, a transcription factor, induces the expression of genes specific for ICs including Jag1, and V-ATPase subunits. Jag1, in turn, inhibits intercalated cell Notch signaling but promotes PC Notch signaling. Tfcp211 deficiency deletes ICs, Jag1 inactivation leads to loss of IC and PC identities. Tfcp211 causes the mixed IC-PC cells. Tfcp2I1 mediates the IC and $\mathrm{PC}$ formation by both cell-autonomous and cell non-autonomous mechanisms. (12). Therefore, Tfcp2I1 coordinates IC and PC development.

Disruptor of telomeric silencing (Dot1) and its mammalian homologues (Dot1l) encodes a methyltransferase specific for histone H3K79, which regulates development, transcription, differentiation and proliferation. Dot1l is expressed in both PCs and ICs (13). Mice with deficient Dot1l in PCs (AQP2-Cre mice were used) had fewer PCs but more intercalated cells, in association with polyuria and lower urine osmolarity. As deletion of Dot11 in PC led to abolished histone H3K79 methylation in PC and undetectable di-methyl K79 in IC, they suggest that AQP2 positive PCs give rise to ICs. CAII deletion leads to decreases in ICs and increases in PCs in the collecting duct (14).

Using AQP2-Cre mice and R26R-EYFP transgenic mice, Kim et al. found that AQP2-positive cells can be differentiated into ICs when fed with low potassium diet (15). Single-cell RNA-seq identified a small fraction of hybrid cells expressing AQP2 (PC marker) and AE1 or pendrin transcripts (IC marker) and suggested signaling crosstalk among the three cell types (16).

Although Notch signaling pathway is important for PC and IC cell fate determination with the collecting duct during development, whether Notch signaling is required to maintain cell type homeostasis in the collecting duct in adult kidney, or whether it is involved in the pathogenesis of nephrotic diabetes insipidus and alteration of PC versus IC ratio, induced by lithium in patients, are still unclear. A recent study published in $\mathcal{F} A S N$ by Mukherjee et al. has largely addressed these issues (17).

They used mice with inducible deletion of both Notch1 and Notch 2 after 3 weeks of age. Elf5 is an early marker specific for PC lineage that regulates AQP2 and AVPR2 
gene expression (18). They used Elf5-rtTA; Tet-OCre;Rosa ${ }^{\text {tdTomato }}$ with or without Hes $1^{\mathrm{f} / \mathrm{f}}$ mice and treated these mice with doxycycline from 3 to 5 weeks of age to turn on tdTomato expression in the Elf5-expressing collecting duct cells to trace cell lineage. They found: (I) in the collecting duct of adult mouse kidney, Notch signaling is evident in both ICs and PCs; (II) mastermindlike-1 (Maml) is an inhibitor of Notch signaling. Mice with induction of dominant-negative Maml in nephrons and collecting duct in 3-4 weeks old mice had decreased Notch signaling and decreased PC genes including Aqp2, Aqp4, and Avpr2 but increased IC genes including Foxil and Slc4a9, in association with increased ICs and decreased PCs and inability to concentrate urine; (III) inducible, conditional inactivation of Notch 1 and Notch2 in mature kidney led to decreases in both the number of the PC and the specific gene of the PC (AQP2) and increases in both the gene of the IC (Foxil and V-ATPase) and the number of the IC number in collecting duct, in association with decreased urine concentrating ability; (IV) Notch signaling activates HES1 expression, and HES1 in turn target Notch ligand such as DLL1, Jag1, and neutrogenin-2. HES1 has been shown to influence the differentiation decision of cells. Inducible, selective deletion of HES1 in nephrons and collecting duct in adult mice led to decreases in both the number of the PC and the specific gene of the PC (AQP2) and increases in both the gene of the IC (Foxi1 and V-ATPase) and the number of the IC number in collecting duct, in association with decreased urine concentrating ability; (V) either Notch $1 \& 2$ deletion or HES1 deletion increased B-ICs in the cortex and A-ICs in the medulla; (VI) Notch signaling is essential for AQP2 expressing cells in the connecting tubule of distal nephron; (VII) fate tracing of mature PCs in the inner medullary collecting duct reveals conversion to ICs with HES1 deletion, and to lesser extent, with lithium treatment; and finally, (VIII) PC to IC conversion due to Hes1 inhibition is related to Foxi1 upregulation. Therefore, these studies clearly indicate that Notch signaling is important in maintaining epithelial cell type homeostasis in collecting ducts in adult kidneys.

\section{Acknowledgments}

Funding: This work was supported by NIH grants, DK51265, DK95785 and DK62794 and the Vanderbilt O'Brien Center (P30DK114809), and the Vanderbilt Center for Kidney Disease.

\section{Footnote}

Conflicts of Interest: The author has no conflicts of interest to declare.

Ethical Statement: The author is accountable for all aspects of the work in ensuring that questions related to the accuracy or integrity of any part of the work are appropriately investigated and resolved.

\section{References}

1. Seely JC. A brief review of kidney development, maturation, developmental abnormalities, and drug toxicity: juvenile animal relevancy. J Toxicol Pathol 2017;30:125-33.

2. Mathew $S$, Chen $X$, Pozzi A, et al. Integrins in renal development. Pediatr Nephrol 2012;27:891-900.

3. Zoetis T, Hurtt ME. Species comparison of anatomical and functional renal development. Birth Defects Res B Dev Reprod Toxicol 2003;68:111-20.

4. Márquez MG, Cabrera I, Serrano DJ, et al. Cell proliferation and morphometric changes in the rat kidney during postnatal development. Anat Embryol (Berl) 2002;205:431-40.

5. Roy A, Al-bataineh MM, Pastor-Soler NM. Collecting duct intercalated cell function and regulation. Clin J Am Soc Nephrol 2015;10:305-24.

6. Zhang MZ, Sanchez Lopez P, McKanna JA, et al. Regulation of cyclooxygenase expression by vasopressin in rat renal medulla. Endocrinology 2004;145:1402-9.

7. Zhang MZ, Wang S, Wang Y, et al. Renal Medullary Interstitial COX-2 (Cyclooxygenase-2) Is Essential in Preventing Salt-Sensitive Hypertension and Maintaining Renal Inner Medulla/Papilla Structural Integrity. Hypertension 2018;72:1172-9.

8. Klein T, Bischoff R. Active metalloproteases of the A Disintegrin and Metalloprotease (ADAM) family: biological function and structure. J Proteome Res 2011;10:17-33.

9. Guo Q, Wang Y, Tripathi P, et al. Adam10 mediates the choice between principal cells and intercalated cells in the kidney. J Am Soc Nephrol 2015;26:149-59.

10. Jeong HW, Jeon US, Koo BK, et al. Inactivation of Notch signaling in the renal collecting duct causes nephrogenic diabetes insipidus in mice. J Clin Invest 2009;119:3290-300.

11. Blomqvist SR, Vidarsson H, Fitzgerald S, et al. Distal 
renal tubular acidosis in mice that lack the forkhead transcription factor Foxi1. J Clin Invest 2004;113:1560-70.

12. Werth M, Schmidt-Ott KM, Leete T, et al. Transcription factor TFCP2L1 patterns cells in the mouse kidney collecting ducts. Elife 2017;6. doi: 10.7554/eLife.24265.

13. Wu H, Chen L, Zhou Q, et al. Aqp2-expressing cells give rise to renal intercalated cells. J Am Soc Nephrol 2013;24:243-52.

14. Breton S, Alper SL, Gluck SL, et al. Depletion of intercalated cells from collecting ducts of carbonic anhydrase II-deficient (CAR2 null) mice. Am J Physiol 1995;269:F761-74.

15. Kim WY, Nam SA, Choi A, et al. Aquaporin 2-labeled cells differentiate to intercalated cells in response to

Cite this article as: Zhang MZ. Notch signaling is essential in collecting duct epithelial cell fate determination during development and maintenance of cell type homeostasis in adult. Ann Transl Med 2019;7(Suppl 8):S376. doi: 10.21037/ atm.2019.12.121 potassium depletion. Histochem Cell Biol 2016;145:17-24.

16. Chen L, Lee JW, Chou CL, et al. Transcriptomes of major renal collecting duct cell types in mouse identified by single-cell RNA-seq. Proc Natl Acad Sci U S A 2017;114:E9989-98.

17. Mukherjee M, deRiso J, Otterpohl K, et al. Endogenous Notch Signaling in Adult Kidneys Maintains SegmentSpecific Epithelial Cell Types of the Distal Tubules and Collecting Ducts to Ensure Water Homeostasis. J Am Soc Nephrol 2019;30:110-26.

18. Grassmeyer J, Mukherjee M, deRiso J, et al. Elf5 is a principal cell lineage specific transcription factor in the kidney that contributes to Aqp2 and Avpr2 gene expression. Dev Biol 2017;424:77-89. 